\title{
The effects of treadmill or overground walking training program on gait in Parkinson's disease
}

\author{
O. Bello, J.A. Sanchez, V. Lopez-Alonso, G. Márquez, L. Morenilla, X. Castro, M. \\ Giraldez, D. Santos-García, M. Fernandez-del-Olmo
}

\begin{abstract}
Background. Gait impairment in Parkinson's disease (PD) patients is characterized by the inability to generate appropriate stride length. Treadmill training has been proposed as a therapeutic tool for PD patients. However, it remains unknown whether treadmill training effects are different from overground walking training. Thus, our goal was to explore the effects of two training programs, walking on a treadmill and walking overground, in PD patients. Methods. $22 \mathrm{PD}$ patients were randomly assigned to a treadmill or overground training group. The training program consisted of 5 weeks ( 3 sessions/week). Before and after the program we evaluated gait kinematics during walking at preferred and maximal speed; Timed Up and Go (TUG); static posturography and knee extensors strength. Gait parameters were reevaluated in the treadmill training group one month after the cessation of the training.

Results. Preferred speed walking improved in both groups after the training program. The treadmill training program, but not the overground, led to an improvement in the stride length at the preferred and maximal walking speed in the PD patients. In addition, the treadmill training group showed improvement of the TUG and static posturography tests. The improvement in gait parameters was maintained one month after the cessation of the treadmill training.

Conclusions. This study provides evidence of a specific therapeutic effect of treadmill training on Parkinsonian gait and balance. Walking on a treadmill may be used as an easy, effective and accessible way to improve the stride length and balance in PD patients.
\end{abstract}

Keywords

Parkinson; Treadmill; Balance; Gait; Exercise

\section{Introduction}

Gait disturbances are one of the principal and most incapacitating symptoms of Parkinson's disease (PD). PD gait is characterized by the inability to generate appropriate stride length [1]. Associated disturbances include a forward-flexed trunk, inadequate flexion at the ankle and knee, insufficient heel strike, reduced arm swing, postural instability, asymmetric stride times for lower limbs and high stride-tostride variability [2], [3], [4], [5], [6] and [7].

Gait impairment in Parkinson's disease (PD) patients is characterized by the inability to generate appropriate stride length [1]. Improvement of gait represents the main goal in rehabilitation and exercise interventions in PD patients. However, due to the age-associated progressive loss of physical function it is difficult to elucidate which rehabilitation approach has a specific impact in PD rather than a generalized benefit from exercise. For example, several studies have shown that rehabilitation in PD patients can result in an improvement of the preferred walking speed but does not enhance stride length [8].

In the last 10 years there has been an increased interest in investigating treadmill training as a potential therapeutic tool for PD patients [9], [10], [11] and [12]. Toole et al. [10] showed an improvement in the gait and balance in PD patients after a 6-week treadmill walking program. Enhancement in the gait rhythmicity and improvements in the motor signs have also been reported. In addition, several studies show that one single session with the treadmill can be effective in improving the gait parameters in PD subjects [13], [14] and [15].

One hypothesis to explain the therapeutic effect of the treadmill suggests that the benefits may be related to proprioceptive sensory cues associated with the belt movement of the treadmill [16]. However, it remains unknown whether the benefits associated with treadmill training are related to the treadmill itself or whether they are simply related to the amount of walking practice that is afforded through treadmill training [17]. Thus, the goal of this study was to explore the effects of two training programs in 
PD patients: treadmill walking and overground walking. Both programs had the same intensity and volume of training. We evaluated several motor tasks such as gait, balance and knee extensors strength before and after each training program. We hypothesized that treadmill training, but not overground walking, would lead to motor improvements in the PD patients.

\section{Methods}

Twenty-two patients with PD (13 males and 9 females, mean age $58.72 \pm 10.35)$ were recruited from the local community and local Parkinson's disease Association, under the supervision of a neurologist and physical therapist. Nineteen patients were in a mild state (range of H\&Y from I to II) and three in a moderate state (III of H\&Y). PD patients were excluded if they had a past history of neurological conditions other than PD, orthopedic, or visual disturbance that affected walking ability. Cardiopulmonary parameters were tested using a graded exercise test (with monitoring of ECG and blood pressure) on the treadmill. Patients that presented signs of cardiovascular or autonomic dysfunction were excluded from the study. A fundamental requirement for inclusion in the study was the ability to walk for 10 min without stopping, walking aids or assistance. All tests were carried out while the patients were ON medication, corroborated by a neurologist and consistently done at the same time of day for each patient. The level of functional disability was determined using the Unified Parkinson's Disease Rating Scale (UPDRS) and the Hoehn and Yahr (H\&Y) scale. The dominant symptoms were tremor, akinetic-rigid and mixed phenotype in 3, 12 and 7 patients, respectively. No patient showed dementia as assessed by a minimental state examination (MMSE > 26) and all of the patients provided their written informed consent to participate in this study. The local ethic committee of University of A Coruña approved the experimental protocol. Details of the subjects are shown in Table 1. 
Table 1. Clinical characteristics of PD subjects. Details of Parkinson's disease patient characteristics.

\begin{tabular}{|c|c|c|c|c|c|c|c|c|}
\hline Patient number & $\begin{array}{l}\text { Age } \\
\text { (years) }\end{array}$ & Sex & $\begin{array}{l}\text { Height } \\
(\mathrm{m})\end{array}$ & $\begin{array}{c}\text { Disease } \\
\text { duration } \\
\text { (years) }\end{array}$ & Type & $\mathrm{H} \& \mathrm{Y}$ & $\begin{array}{l}\text { UPDRS } \\
\text { motor }\end{array}$ & Medication per day (mg) \\
\hline \multicolumn{9}{|c|}{ Treadmill training patients } \\
\hline 1 & 66 & $\mathrm{~F}$ & 1.60 & 12 & $\mathrm{~T}$ & 2.5 & 12 & $\begin{array}{l}\text { Levodopa/Carbidopa 200/50, Levodopa/Benserazide } \\
\text { 550/137.5, Rotigotine 6, Rasagiline 1, Amantadine } 200\end{array}$ \\
\hline 2 & 79 & $\mathrm{~F}$ & 1.49 & 7 & AR & 3 & 35 & Levodopa/Carbidopa 400/50, Pramipexole 0.18 \\
\hline 3 & 58 & M & 1.76 & 6 & M & 2 & 23 & Levodopa/Carbidopa 500/125, Rasagiline 1, Rotigotine 4 \\
\hline 4 & 60 & M & 1.69 & 6 & $\mathrm{AR}$ & 2 & 12 & $\begin{array}{l}\text { Levodopa/Carbidopa 800/200, Entacapone 800, Pramipexole } \\
3.15\end{array}$ \\
\hline 5 & 60 & M & 1.68 & 7 & M & 2 & 13 & $\begin{array}{l}\text { Levodopa/Carbidopa 500/125, Ropinirole 12, Trihexyphenidyl } \\
2\end{array}$ \\
\hline 6 & 62 & $\mathrm{~F}$ & 1.59 & 2 & $\mathrm{AR}$ & 3 & 31 & $\begin{array}{l}\text { Levodopa/Carbidopa 600/150, Entacapone 600, Rotigotine } 6 \text {, } \\
\text { Pramipexole } 3.15\end{array}$ \\
\hline 7 & 68 & M & 1.65 & 1 & $\mathrm{AR}$ & 2.5 & 16 & Levodopa/Carbidopa 150/37.5, Entacapone 600, Rasagiline 1 \\
\hline 8 & 50 & M & 1.70 & 5 & AR & 2 & 19 & Levodopa/Carbidopa 300/75, Rasagiline 1, Rotigotine 8 \\
\hline 9 & 67 & M & 1.80 & 3 & M & 2 & 15 & $\begin{array}{l}\text { Levodopa/Benserazide 500/125, Rasagiline 1, Pramipexole } \\
2.64\end{array}$ \\
\hline 10 & 39 & $\mathrm{~F}$ & 1.63 & 1 & $\mathrm{~T}$ & 2 & 18 & Levodopa/Carbidopa 375/93.75, Entacapone 600, Rasagiline 1 \\
\hline 11 & 45 & M & 1.74 & 3 & M & 2 & 11 & $\begin{array}{l}\text { Levodopa/Carbidopa 225/56.25, Entacapone 600, Rasagiline } \\
\text { 1, Rotigotine } 4\end{array}$ \\
\hline Mean & 59.45 & & 1.66 & 4.82 & & 2.27 & 18.64 & \\
\hline SD & 11.32 & & 0.08 & 3.28 & & 0.41 & 7.99 & \\
\hline \multicolumn{9}{|c|}{ Overground training patients } \\
\hline 1 & 55 & M & 1.72 & 6 & M & 2 & 23 & $\begin{array}{l}\text { Levodopa/Carbidopa 225/56.25, Entacapone } 600 \text {, Rasagiline } \\
\text { 1, Ropinirole } 20\end{array}$ \\
\hline 2 & 56 & M & 1.65 & 2 & $\mathrm{AR}$ & 1.5 & 12 & Pramipexole 2.1 \\
\hline 3 & 51 & $\mathrm{~F}$ & 1.68 & 5 & $\mathrm{AR}$ & 2 & 36 & Levodopa/Benserazide $600 / 150$ \\
\hline 4 & 46 & $\mathrm{~F}$ & 1.61 & 6 & M & 2.5 & 23 & Levodopa/Carbidopa 150/37.5, Entacapone 600, Rasagiline 1 \\
\hline 5 & 46 & $\mathrm{~F}$ & 1.62 & 1 & AR & 2 & 23 & $\begin{array}{l}\text { Levodopa/Carbidopa 150/37.5, Rasagiline 1, Pramipexole } \\
3.15\end{array}$ \\
\hline 6 & 62 & M & 1.73 & 4 & $\mathrm{AR}$ & 2 & 12 & $\begin{array}{l}\text { Levodopa/Carbidopa 150/37.5, Entacapone 600, Pramipexole } \\
3.15\end{array}$ \\
\hline 7 & 61 & $\mathrm{~F}$ & 1.57 & 6 & M & 2 & 23 & Levodopa/Carbidopa 500/50, Pramipexole 3.15 \\
\hline 8 & 63 & M & 1.74 & 8 & $\mathrm{AR}$ & 2 & 21 & $\begin{array}{l}\text { Levodopa/Carbidopa 750/187.5, Entacapone 1000, Rasagiline } \\
\text { 1, Pramipexole } 3.15\end{array}$ \\
\hline 9 & 78 & M & 1.62 & 9 & $\mathrm{AR}$ & 3 & 38 & $\begin{array}{l}\text { Levodopa/Carbidopa 400/100, Levodopa/Benserazide } \\
\text { 700/175, Ropinirole } 20\end{array}$ \\
\hline 10 & 54 & $\mathrm{~F}$ & 1.57 & 6 & AR & 2.5 & 25 & Levodopa/Carbidopa 400/100, Entacapone 800, Rotigotine 12 \\
\hline 11 & 66 & M & 1.67 & 1.5 & $\mathrm{~T}$ & 1 & 7 & Levodopa/Carbidopa 375/37.5, Rasagiline 1 \\
\hline Mean & 58.00 & & 1.65 & 4.95 & & 2.05 & 22.09 & \\
\hline SD & 9.38 & & 0.06 & 2.59 & & 0.52 & 9.44 & \\
\hline $\begin{array}{l}p \text { value between } \\
\text { groups }\end{array}$ & 0.74 & & 0.63 & 0.91 & & 0.42 & 0.36 & \\
\hline
\end{tabular}




\subsection{Procedure}

The patients were randomly assigned to a treadmill training group (Gtreadmill) or overground group (Goverground). All patients were evaluated before (T0) and after the training program (T1). In addition, gait evaluation was repeated in the Gtreadmill group one month later (T2), since this group showed a significant improvement in the stride length after the training program. During this month the patients did not change their daily activities. The training program consisted of 5 weeks, 3 session/week of walking on a treadmill (Gtreadmill group) or walking overground (Gground). In the first week, each session consisted of 4 bouts of 4 min of walking, with 3 min rest between bouts. Each week an additional 4 min bout was added. The walking speed during the training sessions remained constant and was determined as the individual preferred speed obtained for each subject during the first evaluation. Patients from the Gtreadmill group were asked to walk on a treadmill (SporsArt 6300, Sports Arts Fitness) without body weight support, wearing a safety harness to prevent falls. In addition, all patients were asked to hold on to the handrails of the treadmill regardless of whether they needed to or not. All patients were able to walk on the treadmill at their overground comfortable speed from the first block of the first session. The training of the Gground group was conducted in an indoor facility $60 \mathrm{~m}$ long and $10 \mathrm{~m}$ wide. In order to control the walking speed of the Ground patients, each patient wore an MP3 device that provided auditory cues. Between each auditory cue the patients had to walk a distance of $10 \mathrm{~m}$. To provide feedback to the patients regarding their speed, cones were located on the side of the walkway each $10 \mathrm{~m}$. At the moment of each auditory cue, the patient had to arrive to the cone. The pace of the auditory cues was adjusted to the preferred speed of each patient. In a pilot study we determined that the auditory cues did not affect any of the gait parameters in the patients. The walking speed of each patient was monitored in each of the training sessions in order to confirm that the patient was walking at the desired speed.

Before the initial evaluation, all the patients were familiarized with the test for an duration of a week in order to avoid a possible learning effect. All testing and training sessions were carried out while the patients were $\mathrm{ON}$ medication.

\subsection{Tasks}

\subsubsection{Gait evaluation tests}

The patients performed the following tests in the following order: (i) walking overground for 4 min at their preferred speed; (ii) walking overground for $10 \mathrm{~m}$ at maximal speed. All gait tests were performed in the indoor facility described previously. In addition, the patients walked for 4 min on a treadmill at their preferred speed (the same speed that was obtained for overground walking) before the training program (T0). Speed (m/s), cadence (steps/min) and stride length $(\mathrm{m})$ were recorded using force sensitive switches placed in the patient's shoe and synchronized with photocells (BTS SMART Analyzer system) that were placed each $30 \mathrm{~m}$ across the walkway.

\subsubsection{Timed Up and Go (TUG)}

Patients were seated on a chair, and were instructed to stand up, walk at their own comfortable and safe walking speed for $3 \mathrm{~m}$, turn, come back, and sit down on the chair again. We recorded the duration of the following parameters: time to stand up, time to walk $3 \mathrm{~m}$, time to turn, time to return $3 \mathrm{~m}$, time to sit down, and the total time of all the variables. These parameters were recorded using a digital system developed in our laboratory.

\subsubsection{Static posturography}

The area of the center of pressure $(\mathrm{CoP})$ was recorded for the quiet stance using a stabilometric platform (Kistler 9286BA) in four different randomized conditions: eyes opened and closed, during and in the absence of performing a cognitive task. The subjects' feet were placed in parallel and spaced $10 \mathrm{~cm}$ apart. One $60 \mathrm{~s}$ trial was recorded for each condition. During the balance tests the patients were instructed to keep a stance position. For the dual-task condition patients were asked to keep a stance position and to perform the additional cognitive task. No instructions for priority of one of the tasks were given (stand vs. cognitive task). In the cognitive task condition the patients were required to listen to one of the researchers reading a text and at the end report the number of times that two different words, previously reported, were repeated. To quantify the cognitive task we assigned 1 point for each correct recall of a word. 


\subsubsection{Knee extensors strength testing}

For the isometric knee extension force tests, the patients were placed in a seated position on an isometric knee extensor machine (Leg Extension MED, Technogym Trading S.A.) and were securely strapped into the test chair. The chair had a long backrest, providing full back and head support. The hip and knee were at $90^{\circ}$ of flexion (a $0^{\circ}$ knee extension refers to a horizontal leg-thigh position). After a warm-up we recorded two trials of maximal voluntary knee force extensions performed with both legs simultaneously, two trials with the right leg and two trials with left leg.

\subsection{Statistic analysis}

ANOVA of repeated measures was conducted with Group (Gtreadmill and Gground) and Time (T0, and T1) as factors for the following variables: speed, stride length and cadence for the gait tasks; time to stand up, time to walk $3 \mathrm{~m}$, time to turn, time to return $3 \mathrm{~m}$, time to sit down and the total time for the TUG test; area of CoP during posturography tests; and maximal voluntary contraction during the knee extensor strength test.

Wilcoxon test was performed in the comparison of the cognitive task score during the posturography tests and in the comparison of the motor section score of the UPRDS, between T0 and T1 in each group.

$T$ tests were conducted in the comparison of the gait parameters in the Gtreadmill group between T0 and $\mathrm{T} 2$.

All statistical analyses were performed using SPSS (SPSS, Chicago, IL). A $p$ value $\leq 0.05$ was considered statistically significant.

\section{Results}

Both groups were homogeneous in all the variables analyzed since there were no significant differences between the groups at $\mathrm{T} 0$ (Table 2). 
Table 2. Training effects on the tasks evaluated.

\begin{tabular}{|c|c|c|c|c|c|}
\hline \multirow[t]{3}{*}{ Item } & \multicolumn{5}{|l|}{ Group } \\
\hline & \multicolumn{2}{|l|}{ Gground } & \multicolumn{3}{|l|}{ Gtreadmill } \\
\hline & T0 & $\mathrm{T} 1$ & T0 & $\mathrm{T} 1$ & $\mathrm{~T} 2$ \\
\hline \multicolumn{6}{|l|}{ UPDRS } \\
\hline \multirow[t]{2}{*}{ Motor score } & $22.09 \pm 2.84$ & $18.2 \pm 2.01$ & $18.64 \pm 2.4$ & $21.27 \pm 2.72$ & \\
\hline & \multicolumn{3}{|c|}{ Walking at preferred speed } & & \\
\hline Speed $(\mathrm{m} / \mathrm{s})$ & $1.33 \pm 0.07$ & $1.39 \pm 0.08^{*}$ & $1.26 \pm 0.09$ & $1.36 \pm 0.09^{*}$ & $1.37 \pm 0.11^{*}$ \\
\hline Cadence (steps/min) & $117.98 \pm 3.52$ & $120.69 \pm 3.54^{*}$ & $118.59 \pm 2.77$ & $121.58 \pm 2.74^{*}$ & $123.47 \pm 2.73^{*}$ \\
\hline Stride length $(\mathrm{m})$ & $1.35 \pm 0.05$ & $1.38 \pm 0.05$ & $1.27 \pm 0.08$ & $1.33 \pm 0.07^{*}$ & $1.33 \pm 0.08^{*}$ \\
\hline \multicolumn{6}{|l|}{ Walking at maximal speed } \\
\hline Speed $(\mathrm{m} / \mathrm{s})$ & $1.78 \pm 0.1$ & $1.76 \pm 0.07$ & $1.63 \pm 0.14$ & $1.69 \pm 0.12$ & $1.71 \pm 0.13$ \\
\hline Cadence (steps/min) & $135.85 \pm 4.11$ & $135.74 \pm 2.6$ & $134.02 \pm 4.28$ & $135.4 \pm 3.18$ & $136.88 \pm 3$ \\
\hline Stride length (m) & $1.56 \pm 0.05$ & $1.55 \pm 0.05$ & $1.44 \pm 0.09$ & $1.49 \pm 0.09^{*}$ & $1.52 \pm 0.11^{*}$ \\
\hline \multicolumn{6}{|c|}{ Walking on treadmill at initial preferred speed } \\
\hline Speed $(\mathrm{m} / \mathrm{s})$ & $1.33 \pm 0.07$ & $1.33 \pm 0.07$ & $1.26 \pm 0.09$ & $1.26 \pm 0.09$ & \\
\hline Cadence (steps/min) & $118.8 \pm 4.34$ & $118.8 \pm 4.7$ & $117.6 \pm 3.62$ & $117.6 \pm 4.34$ & \\
\hline Stride length $(\mathrm{m})$ & $1.36 \pm 0.05$ & $1.35 \pm 0.07$ & $1.29 \pm 0.03$ & $1.28 \pm 0.07$ & \\
\hline \multicolumn{6}{|l|}{$T U G$} \\
\hline Total time (s) & $10.17 \pm 0.45$ & $10.30 \pm 0.68$ & $12.87 \pm 1.73$ & $11.26 \pm 1.07^{*}$ & \\
\hline Time to stand up (s) & $1.04 \pm 0.09$ & $1.11 \pm 0.12$ & $1.52 \pm 0.32$ & $1.10 \pm 0.16^{*}$ & \\
\hline Time to walk (s) & $2.20 \pm 0.08$ & $2.13 \pm 0.11$ & $2.53 \pm 0.3$ & $2.44 \pm 0.21$ & \\
\hline Time to turn (s) & $2.62 \pm 0.16$ & $2.57 \pm 0.19$ & $3.23 \pm 0.44$ & $2.76 \pm 0.27^{*}$ & \\
\hline Time to back (s) & $2.16 \pm 0.08$ & $2.18 \pm 0.11$ & $2.70 \pm 0.39$ & $2.43 \pm 0.19$ & \\
\hline Time to sit down (s) & $2.14 \pm 0.12$ & $2.31 \pm 0.18$ & $2.89 \pm 0.4$ & $2.53 \pm 0.29^{*}$ & \\
\hline \multicolumn{6}{|l|}{ Posturography } \\
\hline Eyes open $\left(\mathrm{mm}^{2}\right)$ & $3356 \pm 432$ & $2707 \pm 421$ & $2599 \pm 313$ & $3013 \pm 454$ & \\
\hline Eyes close $\left(\mathrm{mm}^{2}\right)$ & $4143 \pm 621$ & $3210 \pm 405$ & $3709 \pm 355$ & $4101 \pm 436$ & \\
\hline $\begin{array}{l}\text { Eyes open and cognitive task } \\
\left(\mathrm{mm}^{2}\right)\end{array}$ & $2514 \pm 361$ & $2421 \pm 476$ & $2726 \pm 253$ & $3156 \pm 543$ & \\
\hline $\begin{array}{l}\text { Eyes close and cognitive task } \\
\left(\mathrm{mm}^{2}\right)\end{array}$ & $3813 \pm 548$ & $2992 \pm 455^{*}$ & $3050 \pm 252$ & $3882 \pm 555^{*}$ & \\
\hline $\begin{array}{l}\text { Cognitive task with eyes open } \\
\text { (score) }\end{array}$ & $1 \pm 0.2$ & $1 \pm 0.25$ & $0.82 \pm 0.23$ & $1.09 \pm 0.21$ & \\
\hline $\begin{array}{l}\text { Cognitive task with eyes close } \\
\text { (score) }\end{array}$ & $1.40 \pm 0.21$ & $1.40 \pm 0.21$ & $0.82 \pm 0.18$ & $1.45 \pm 0.25^{*}$ & \\
\hline \multicolumn{6}{|l|}{ Knee extensor } \\
\hline Both legs $(\mathrm{N})$ & $1261 \pm 145$ & $1237 \pm 124$ & $1344 \pm 189$ & $1332 \pm 179$ & \\
\hline Affected leg (N) & $650 \pm 106$ & $578 \pm 72$ & $817 \pm 138$ & $804 \pm 129$ & \\
\hline Non-affected leg $(\mathrm{N})$ & $649 \pm 83$ & $573 \pm 62$ & $863 \pm 141$ & $841 \pm 136$ & \\
\hline
\end{tabular}

T0 (evaluation before training program), T1 (evaluation after training program), and T2 (evaluation one month after training program).

* Significant differences with T0 $(p \leq 0.05)$. Note that no differences were found between groups at T0. 
The total walking time of the program was 360 min for each patient.

The ANOVA showed a significant effect of Time factor during the 4 min walking, for the speed $(F=19.84, p<0.001)$ and cadence $(F=17.47, p<0.001)$, without a significant group effect or Group $\times$ Time interaction. Both speed and cadence increased significantly in both groups after the training program. However, the ANOVA showed a significant effect of Time $(F=13.92, p<0.01)$ and Group $\times$ Time interaction $(F=4.48, p<0.05)$ for the stride length. Post hoc analysis indicated that stride length only increased significantly $(p<0.05)$ in the treadmill group, and not in the overground group. The stride length increased by $5.70 \%$ after the training program, and was significantly larger compared to the $1.71 \%$ increase that was observed in the Gground group ( $p<0.047)$.

For the test in which patients walked $10 \mathrm{~m}$ at maximal speed, we did not find significant differences in the cadence and speed before and after the training programs. For the stride length the ANOVA showed a significant Group $\times$ Time interaction $(F=5.53, p=0.029)$. Post hoc analysis showed a significant increase of stride length in the treadmill group after the treadmill training program $(p=0.010)$ but no significant differences before and after training in the overground group. The increase in the treadmill group was of $3.46 \%$, which was significantly larger than in the overground group $(-0.60 \%, p=0.04)$.

The improvements in gait for both the 4 min and maximal speed walking tests were maintained in the Gtreadmill group one month after the end of the training program. Fig. 1 illustrates the average gait parameters for gait at the preferred speed and gait at the maximal speed for all the patients.
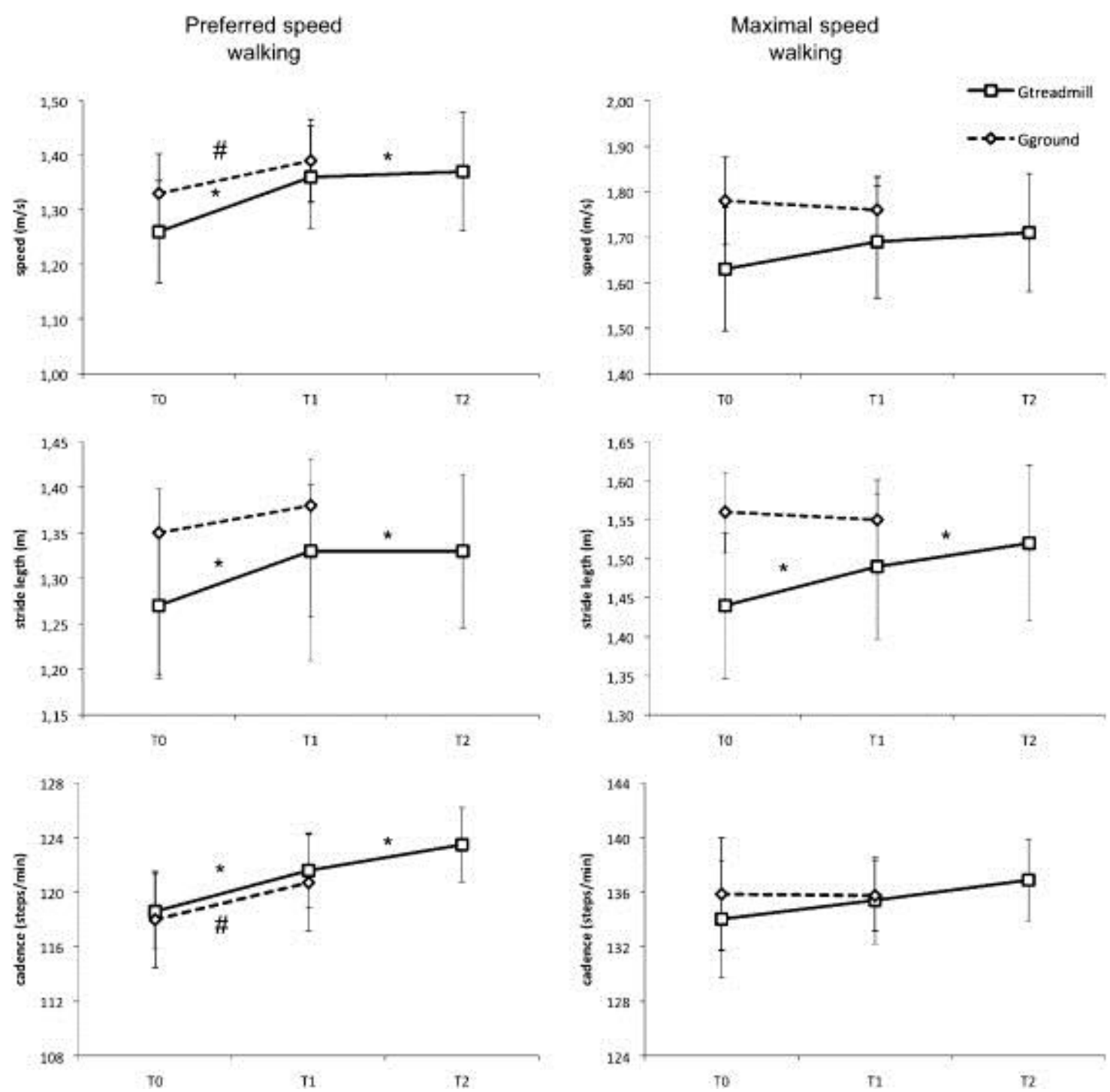

Fig. 1. Training effects on gait measures. T0 (evaluation before training program), T1 (evaluation after training program), and T2 (evaluation one month after training program). * Significant differences with T0 $(p \leq 0.05)$ in the treadmill training group. \# Significant differences with T0 $(p \leq 0.05)$ in the overground training group. 
For the TUG test the ANOVA showed significant Group $\times$ Time interactions for the total time to complete the test $(F=5, p=0.05)$, time to stand-up $(F=5.64, p=0.02)$, turn $(F=6.45, p=0.01)$ and sit down $(F=9.99, p=0.005)$. The post hoc showed a significant decrease $(p<0.05)$ in all these parameters in the treadmill group and no significant changes in the overground group.

The ANOVA for the static posturography parameters showed a significant Group $\times$ Time interaction for the area of CoP, only in the condition with eyes closed while performing a cognitive task $(F=4.86$, $p=0.03)$. Post hoc analyses indicate a significant increase in this parameter for the treadmill group $(p=0.03)$ and a significant decrease for the overground group $(p=0.02)$. There was a significant improvement $(p=0.03)$ in the cognitive test score after the training program for the treadmill group, and no significant changes in the overground group. Fig. 2 illustrates CoP measures and provides an example of one subject in each group.

TO

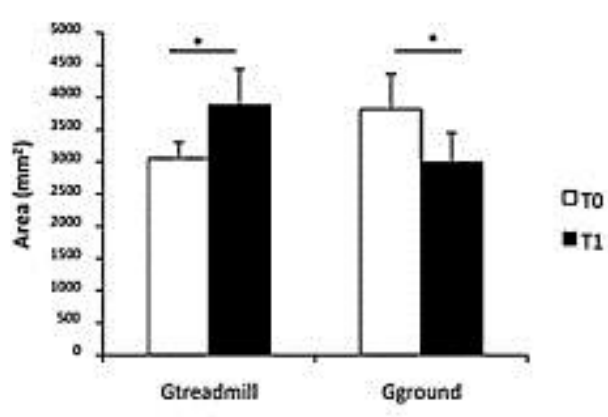

$\mathrm{T} 1$

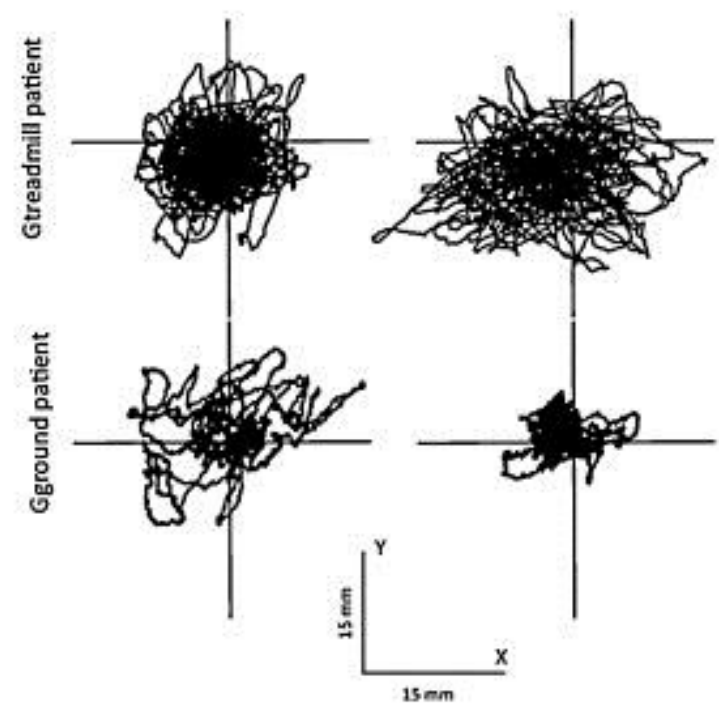

Fig. 2. Training effects on center of pressure $(\mathrm{CoP})$ during quiet stance with eyes closed and during the cognitive task. Left figure shows the changes of the area of $\mathrm{CoP}$ for each group after the training program. Right figure represents the CoP traces in two patients, before and after the treadmill training (upper graphs) or overground training (lower graphs). T0 (evaluation before training program) and T1 (evaluation after training program). * $p \leq 0.05$.

No significant differences were found for the knee extensor strength test.

\section{Discussion}

The main finding of the present study is that a training program consisting of walking on a treadmill, but not overground, led to an improvement in the stride length at the preferred and maximal walking speed in PD patients. These improvements were maintained one month after the cessation of the training.

The gait in Parkinson's disease is affected mainly due to a reduced amplitude of the stride length, while the cadence remains unaffected [1]. In our study, both groups improved their preferred speed after the training programs. The improvement in the overground group was due to an increase in the cadence but not the stride length, suggesting a generalized benefit from exercise. However, the improvement of the stride length that was observed only in the group that trained on the treadmill highlights a specific effect of treadmill training. In the treadmill group, stride length improvements were seen for both preferred and maximal speed of stride length. While the improvement of the stride length at the preferred speed could have resulted from an overall increase in speed, this is unlikely for the maximal gait parameters, since only the maximal stride length increased after the treadmill training and not the maximal speed. Thus, it seems that the treadmill training lead to an increase in stride length and thus to a recovery of a more normal pattern of gait overground. Importantly, the improvement in the stride length of the patients in the treadmill group was only observed once these patients walked overground but not 
apparent while they were walking on the treadmill. This may be due to the mild to moderate state of the disease in the cohort of patients, since a previous study shows that only PD patients in an advanced state (III or higher of H\&Y scale) increased the stride length when walking on the treadmill [13].

One possible explanation for the improvement of the stride length that was observed in the treadmill group may be due to an improvement in the balance of the patients. This is supported by our findings that the patients were able to stand up, turn and sit down faster after the treadmill training. These are all movements that require a large degree of balance. These findings are in line with previous studies that have shown that balance improvements were associated with treadmill training [10], [18] and [19]. Moreover, in our study, during the static balance test, where patients had to perform a cognitive task with closed eyes, only the PD patients that completed the treadmill training were able to increase the area of CoP and improve their performance of the cognitive task. Sway area has been shown to be reduced in PD patients [20], thus our results suggest that the PD patients improved their balance after the treadmill training, allowing them to allocate more attention to the performance of the cognitive task. We speculate that the improvement in balance may allow the patients to project their center of mass forward to a greater extent and as a result improve their gait by increasing their stride length. In addition, the absence of change in the strength of the knee extensor muscles in the patients, rule out a possible peripheral muscle effect as a result of treadmill training.

Several mechanisms have been proposed to explain the improvement in gait induced by treadmill training in PD. For example, the treadmill may provide propioceptive signals, triggering intact circuits and bypassing the defective pallidocortical circuit [13] and increasing corticomotor excitability to induce motor learning [21]. However, it remains unknown how these mechanisms can then lead to enhanced gait overground since in our study the patients did not improve their stride length on the treadmill. In addition, one limitation of this study is the small number of participants. Nevertheless, this study clearly shows, for first time, that the effects associated with treadmill training are related with the treadmill itself and not due to the amount of walking since no effects were found in the overground group.

The treadmill training program used in this study was characterized by a very low intensity and a constant speed that was maintained across sessions. This may explain the fact that the average increase in stride length was only $5.7 \%$, slightly inferior to a previously study using high intensity and incremental speed treadmill training [12]. Thus, it seems that high intensity treadmill training or incremental speed is not necessarily required in order to achieve a significant improvement in gait, which can make the use of treadmill training more accessible even to advanced PD patients with physical fitness disabilities.

The present study provides evidence of a specific therapeutic effect of treadmill training on Parkinsonian gait and balance. This is of relevance since treadmill training can be used as an easy, effective and accessible way to improve the stride length and balance in PD patients.

\section{Funding}

The authors received financial support for the research of this article from the Ministerio de Ciencia e Innovacion, Spain (ref. DEP-2011-22466).

\section{Conflict of interest}

All authors have no conflict of interest and no further financial disclosure to make.

\section{References}

[1] M.E. Morris, R. Iansek, T.A. Matyas, J.J. Summers Stride length regulation in Parkinson's disease. Normalization strategies and underlying mechanisms Brain, 119 (Pt 2) (1996), pp. 551-568

[2] E. Knutsson An analysis of Parkinsonian gait Brain, 95 (1972), pp. 475-486

[3] M.P. Murray, S.B. Sepic, G.M. Gardner, W.J. Downs Walking patterns of men with parkinsonism American Journal of Physical Medicine \& Rehabilitation, 57 (1978), pp. 278-294

[4] S.G. Bowes, P.K. Clark, A.L. Leeman, et al. Determinants of gait in the elderly parkinsonian on maintenance levodopa/carbidopa therapy British Journal of Clinical Pharmacology, 30 (1990), pp. 13-24

[5] O. Blin, A.M. Ferrandez, G. Serratrice Quantitative analysis of gait in Parkinson patients: increased variability of stride length Journal of the Neurological Sciences, 98 (1990), pp. 91-97

[6] E. Ueno, N. Yanagisawa, M. Takami Gait disorders in parkinsonism. A study with floor reaction forces and EMG Advances in Neurology, 60 (1993), pp. 414-418

[7] J.M. Hausdorff, M.E. Cudkowicz, R. Firtion, J.Y. Wei, A.L. Goldberger Gait variability and basal ganglia disorders: stride-to-stride variations of gait cycle timing in Parkinson's disease and Huntington's disease Movement Disorders, 13 (1998), pp. 428-437

[8] G. Kwakkel, C.J. de Goede, E.E. van Wegen Impact of physical therapy for Parkinson's disease: a critical review of the literature Parkinsonism and Related Disorders, 13 (2007), pp. S478-S487 
[9] I. Miyai, Y. Fujimoto, H. Yamamoto, Y. Ueda, T. Saito, S. Nozaki, et al. Long-term effect of body weightsupported treadmill training in Parkinson's disease: a randomized controlled trial Archives of Physical Medicine and Rehabilitation, 83 (2002), pp. 1370-1373

[10] T. Toole, C.G. Maitland, E. Warren, M.F. Hubmann, L. Panton The effects of loading and unloading treadmill walking on balance, gait, fall risk, and daily function in Parkinsonism Neurorehabilitation, 20 (2005), pp. 307322

[11] S. Frenkel-Toledo, N. Giladi, C. Peretz, T. Herman, L. Gruendlinger, J.M. Hausdorff Treadmill walking as an external pacemaker to improve gait rhythm and stability in Parkinson's disease Movement Disorders, 20 (2005), pp. 1109-1114

[12] T. Herman, N. Giladi, L. Gruendlinger, J.M. Hausdorff Six weeks of intensive treadmill training improves gait and quality of life in patients with Parkinson's disease: a pilot study Archives of Physical Medicine and Rehabilitation, 8 (2007), pp. 1154-1158

[13] O. Bello, J.A. Sanchez, M. Fernandez-Del-Olmo Treadmill walking in Parkinson's disease patients: adaptation and generalization effect Movement Disorders, 23 (2008), pp. 1243-1249

[14] I. Miyai, Y. Fujimoto, Y. Ueda, H. Yamamoto, S. Nozaki, T. Saito, et al. Treadmill training with body weight support: its effect on Parkinson's disease Archives of Physical Medicine and Rehabilitation, 81 (2000), pp. 849852

[15] M. Pohl, G. Rockstroh, S. Rückriem, G. Mrass, J. Mehrholz Immediate effects of speed-dependent treadmill training on gait parameters in early Parkinson's disease Archives of Physical Medicine and Rehabilitation, 84 (2003), pp. 1760-1766

[16] O. Bello, G. Márquez, M. Camblor, M. Fernandez-Del-Olmo Mechanisms involved in treadmill walking improvements in Parkinson's disease Gait and Posture, 32 (2010), pp. 118-123

[17] Earhart, Williams Treadmill training for individuals with Parkinson disease Physical Therapy, 97 (2012), pp. 893-897

[18] B. Cakit, M. Saracoglu, H. Genc, H. Erdem, L. Inan The effects of incremental speed-dependent treadmill training on postural instability and fear of falling in Parkinson's disease Clinical Rehabilitation, 21 (2007), pp. 698-705

[19] E.J. Protas, K. Mitchell, A. Williams, H. Qureshy, K. Caroline, E.C. Lai Gait and step training to reduce falls in Parkinson's disease NeuroRehabilitation, 20 (2005), pp. 183-190

[20] M. Schieppati, M. Hugon, M. Grasso, A. Nardone, M. Galante The limits of equilibrium in young and elderly normal subjects and in parkinsonians Electroencephalography and Clinical Neurophysiology, 93 (1994), pp. 286298

[21] T. Herman, N. Giladi, J.M. Hausdorff Treadmill training for the treatment of gait disturbances in people with Parkinson's disease: a mini-review Journal of Neural Transmission, 116 (2009), pp. 307-318 